\title{
BidPacket: trading bandwidth in public spaces
}

\author{
Bernardo A. Huberman ${ }^{1}$ - Sitaram Asur ${ }^{1}$
}

Accepted: 13 September 2016 / Published online: 4 October 2016

() The Author(s) 2016. This article is published with open access at Springerlink.com

\begin{abstract}
Smart devices such as smartphones and tablets are used extensively in public spaces for the transmission and reception of content in the form of text, photos and streaming videos. Since the bandwidth provided for wireless access is limited in public areas, it becomes an issue for users to gain access to the bandwidth they need at the right times. While an omniscient controller could assign bandwidth to each device on the basis of their needs and overall availability, imperfect information about the instantaneous state of the wifi access patterns and needs of users make for a very inefficient allocation of such bandwidth. This paper provides a solution for bandwidth allocation by creating a market among users of smart devices so that they can bid for extra bandwidth when they need it and sell it when they don't. They do so by using a virtual currency that is conserved so that each device owner maximizes his own utility. This utility function is composed of both the benefit accrued from accessing bandwidth and the loss of the currency incurred in bidding for such bandwidth. Extensive simulations show that this market-based method outperforms an omniscient model when demand is uncertain, while minimizing bandwidth consumption.
\end{abstract}

Keywords Bandwidth allocation · Trading market · Virtual currency · Economic models

Bernardo A. Huberman

bahuberman@gmail.com

Sitaram Asur

sitaram.asur@hpe.com

1 Hewlett Packard Labs, Palo Alto, CA 94304, USA 


\section{Introduction}

The advent of smart devices has resulted in unprecedented usage of wifi networks to transmit media content. At the recent Superbowl $2016^{1}$, for example, there were 20,300 maximum concurrent Wi-Fi users at a particular time, and more than 3 gigabits of data per second were carried over the wireless network for four continuous hours. In many public locations such as airports, hotels, stadiums and malls, the amount of bandwidth allocated is becoming constrained due to massive usage demands and the popularity of photo, video and audio streaming apps like Netflix, YouTube, Pandora, Spotify, Instagram and Vine. The hospitality industry has identified this issue and is struggling to offer wifi services that can sustain this demand. ${ }^{2}$

Two possible solutions can mitigate this problem. First, one can augment the amount of available bandwidth by installing increasing numbers of access points, which has the drawback of being costly to the providers and result in over provisioning when user needs fluctuate over time. Second, one can operate with existing installed levels of bandwidth, but provide smart allocations by taking into account the varying needs of the user. While this solution is economically desirable, there remains the problem of dealing with the fluctuating needs of users. In most real-world settings, it is hard to predict demand at an individual device level. Hence an omniscient bandwidth allocation system controller that needs to know user requirements cannot be efficient in such settings.

In this paper we propose a market-based method for resource allocation in a distributed public setting. Rather than having a central controller to allocate bandwidth, we design the system to work on a peer-to-peer level with each device owner bidding for extra bandwidth based on his or her requirements. The market also provides incentives to those owners that are not currently using the bandwidth to sell their quota to others who need it. The resulting system is not only extremely adaptable to unforeseen changes in the data or network failures, but it also makes the overall capacity of the system far lower than in the over-provisioned scenario.

The market transactions are performed using a virtual currency. Each customer is provided an initial budget and a basic bandwidth allocation. If a user wishes to acquire more bandwidth for a certain time interval he can bid using her conserved currency. Other users that are not using their bandwidth can thus sell it for given intervals of time to the buyers and gain currency which can be used later for either buying more bandwidth or obtain discounts at the public place. Each user maximizes his utility function, which takes into account the gain he accrues from the extra bandwidth and the loss incurred in buying bandwidth. In real-world scenarios users can be also entitled to rewards such as hotel points or airline miles based on their transactions in the market.

Finally, we conduct experiments to compare the market-based method with a centralized allocation method. We simulate both scenarios and examine the performance

\footnotetext{
${ }^{1}$ http://www.geekwire.com/2016/super-bowl-data-usage

${ }^{2}$ http://hotelexecutive.com/business_review/3046/hotel-wi-fi-balancing-budget-bandwidth
} 
in transmitting data under various constraints. We observe that the market provides significant benefits when there is uncertain demand for bandwidth, a situation which is representative of many real-world scenarios.

\section{Related work}

Market mechanisms have been applied with great success in various resource allocation applications such as networks, computer system resources, manufacturing etc. [1]. Waldspurger et al. [12] developed a market-based computational system called Spawn for trading idle computational resources in a distributed network of heterogeneous computer workstations. Lai et al. [6] developed Tycoon, another market-based resource allocation system that allowed for users to differentiate the values of their jobs without manual bidding overhead. Mechanical structures and their patterns can also be controlled and stabilized by market-based algorithms, as shown by Guenther et al. [3] and Lynch and Law [7]. Last but not least there is the work on market-based control mechanisms for electric power demand response, as exemplified by Papavasiliou et al. [8].

There is also work on auction-based control of energy resources in a building [5], of spectrum sharing [4] and of traffic lights [2], which points to the utility of markets for decentralized control of many diverse resources.

There has been some work on markets in the context for bandwidth but not for public space trading. For example, Rasmusson and Aurell [11] proposed a model of price dynamics in bandwidth markets and studied how prices equilibrate under many different conditions. Wang et al. [13] developed a market-driven approach to regulate the behavior of selfish nodes that provide or consume services. However, their focus is on reinforcement learning to help selfish nodes to incrementally adapt to the local market, and to make optimized strategic decisions based on past experiences. Our focus is on the design of a marketplace for public spaces where users can trade bandwidth. Paris et al. [9] discuss a market where mobile operators can lease bandwidth from third parties through their access points to increase network capacity. Qiu and Marbach [10] develop a price-based approach to stimulate users to relay data packets for other users.

\section{The bidpacket market}

In this market there are $N$ consumers that are allocated a given amount of bandwidth by a central wifi provider. In a real-world scenario this could be users in a hotel, an airplane, a train, or an airport lounge that are sharing a wifi hotspot.

Apart from the initial allocation of bandwidth, all users are also awarded a budget of virtual currency. This could be done fairly with all users getting the same amount or with certain elite users being awarded more. If the initial bandwidth allocation satisfies the need of the users they start using the bandwidth. At any point in time, if any user wishes to acquire additional bandwidth he can bid to buy more, while users who are not utilizing their quota can decide to sell their bandwidth to others. 
Thus all the profit that sellers get from selling bandwidth can be redistributed to the other consumers when they in turn buy bandwidth. This implies that the total amount of currency and resources in the system will stay constant. In our context, this is the total amount of bandwidth that the producer allocates to the consumers (or money associated with its use).

We assume that the market reaches equilibrium between overall supply and demand, and this equilibrium determines the price and the amount of bandwidth traded. Each consumer gets the amount of bandwidth that he offers to buy for the equilibrium price and uses the bandwidth to consume/upload content in his device for a specified duration.

\subsection{Utility functions for buyers and sellers}

Each consumer as a buyer $i$ buys an amount of bandwidth, $B_{i}$, that depends on his utility function, $U_{i}$ which reflects a tradeoff between the bandwidth he consumes and the loss of wealth involved. There are many utility functions that could be employed for this purpose. A simple one expressed in terms of the price, $p$ and a user $i$ 's wealth $w_{i}$ is given as :

$$
U_{i}=b_{i} B_{i}-\frac{p}{2 w_{i}} B_{i}^{2}
$$

where $b_{i}$ is a weight that can be modulated to determine the need to purchase for user $i$.

The buyer demand function is obtained by maximizing his utility function as a function of the bandwidth, which gives $\frac{w_{i}}{p} b_{i}$.

This demand function causes the consumer to demand more bandwidth when his needs are large $\left(b_{i}\right.$ is large). It also reflects the trade-off involved in maintaining wealth: demand decreases both with increasing price as well as when consumers have little wealth. The overall demand function for the system is just the sum of these individual demands, giving

$$
B^{\text {demand }}=\frac{\sum_{i=1}^{n} w_{i} b_{i}}{p}
$$

Similarly, each user as seller tries to maximize his profit, $\rho$, given by the difference between his revenue from selling bandwidth and his personal cost $C(B)$.

$$
\rho=p B-C(B)
$$

To minimize the total bandwidth used, we select a cost function for which the cost per unit of bandwidth, $\frac{C(B)}{B}$, increases with the amount of bandwidth sold.

A simple example of such a cost function is $C(B)=\frac{B^{2}}{2 a_{i}}$ where the parameter $a_{i}$ reflects the importance of conserving bandwidth and using it for user $i$.

We obtain the producers supply function by maximizing its profit:

$$
\frac{d \rho}{d B}=p-\frac{d C}{d B}=0
$$

which implies $B^{\text {supply }}(p)=a_{i} p$. 
So we obtain the overall supply function as

$$
B^{\text {supply }}=p \sum_{i=1}^{n} a_{i}
$$

From this, the price and amount of traded bandwidh is determined by the point where the overall supply and demand curves intersect, i.e., $B^{\text {supply }}=B^{\text {demand }}$.

For our choices of the utility and cost functions, this condition can be solved analytically to give the equilibrium price as

$$
p^{\text {trade }}=\sqrt{\frac{\sum_{i=1}^{n} w_{i} b_{i}}{\sum_{i=1}^{n} a_{i}}}
$$

\subsection{Incentivizing participation}

One of the issues in deploying markets is providing incentives for users to sell unused bandwidth to those who need it. In the BidPacket market this is done through the use of a virtual currency. Each user is originally awarded a budget. Users attempt to conserve or gain virtual currency in order to obtain rewards from the market makers. Real-world rewards can be in the form of loyalty points in hotels or airline miles.

\section{Implementation of the market}

The market is designed to operate in public spaces such as hotels, airports, resorts, airplanes, etc. using the installed wifi allocation strategies. It can be implemented as a built-in application that manages the market of users and thus it will not impact the underlying layers of the wireless network. Each market will be associated with a wifi hotspot or access point, so that users can only trade with other users using that particular wifi access point.

Each user connects to an app on their smart device that can then be used to buy or sell bandwidth. When a user registers at an access point, he is awarded a budget of virtual currency. The list of users and their currency is maintained at the access point level. Note that the budget is allocated only the first time a user registers using the app with an access point. Depending on the use case, a user's budget can be associated either with only one access point or carried over across different access points. In a hotel for instance, a user would tend to use wifi in their rooms rather than the corridors whereas in an airport, users who move across lounges or from lounge to gate can carry over their identity and currency to other access points. Since the user connects through an app on any smart device that he owns, the identity of the user and the available currency can be maintained across devices.

Bids entered in the system are displayed to other users who are registered. These users can then decide to sell their quota of bandwidth if they are not planning to use it. There is also a time window that is associated with each bid indicating how long the user requires the excess wifi. We use hours as the unit of time that users can bid for or sell. For instance, a user may desire to use higher bandwidth for 2 hours to 
watch a streaming movie. It may be possible for users who are not fulfilling their purchased bandwidth to return or resell some of the bandwidth to other users.

Note that there is some wifi that is always present for a user. This enables the user to transact in the market while also providing functionality for simple low-bandwidth tasks like instant messaging and email. Also if a user is not using bandwidth and is not participating in the market (selling), the bandwidth is redistributed to all the users in the system. This way there is no wastage of bandwidth due to allocation to inactive users.

Having the market at the level of access points also allows the scalability to be defined by the wifi provider. Thus the market does not need to worry about having too many users transacting. It can only allow users who are registered at the access point to trade the wifi. Also the users themselves do not have access to the currency mechanism so there is no possibility of any modifications by users directly.

\section{Experiments}

To validate our assumption that markets can improve efficiency of bandwidth sharing, we simulated a market for bandwidth allocation for a set of $N$ users. We assume an overall bandwidth $B$ which is initially partitioned evenly across the devices. Note that this allocation can also be done unfairly with some owners of devices enjoying higher priorities or access privileges. Each user is also endowed with an initial budget of currency $C$ which will be used for trading purposes. In the simplest scenario, we assume that this initial budget is equal for each user, although for other scenarios this assumption may be relaxed.

At each instant of time, a user chooses to send a file based on a certain probability, which we assume to derive from a uniform distribution. For the purposes of this simulation we assume the file to be a video file which is then modeled as a Gaussian with mean 150 and standard deviation 50 .

If the device decides to send the file at that time instant, it has the option of using the default bandwidth it has been originally assigned $(B / N)$ or purchasing more bandwidth for a certain amount of time in order to send or receive the file at a faster rate. This decision to purchase extra bandwidth is based on a utility function as described earlier. To simulate this utility we use two variables - one modeling the urgency (as a uniform distribution) and the other taking into account the relative wealth (budget) that the device possesses. For instance, if the device is low on currency, it is less likely to purchase bandwidth than if it was wealthy.

If the device chooses to purchase bandwidth, it registers a bid with a corresponding price and a time. The price is calculated to be proportional to the size of the file the device wants to send and its urgency requirement.

Other users who are not sending files can choose to sell their bandwidth and get compensated in the form of currency. Each seller picks the buyer who is offering the highest price among all the bids. Once a sale happens, the seller then relinquishes their bandwidth to the buyer for the duration of the data transfer. The decision to sell is also dependent on the budget possessed by the users, with users having low budget more inclined to sell bandwidth to gain more wealth than others. The buyers can then receive or transmit their data at a higher bitrate and receive a quicker transfer. If a 


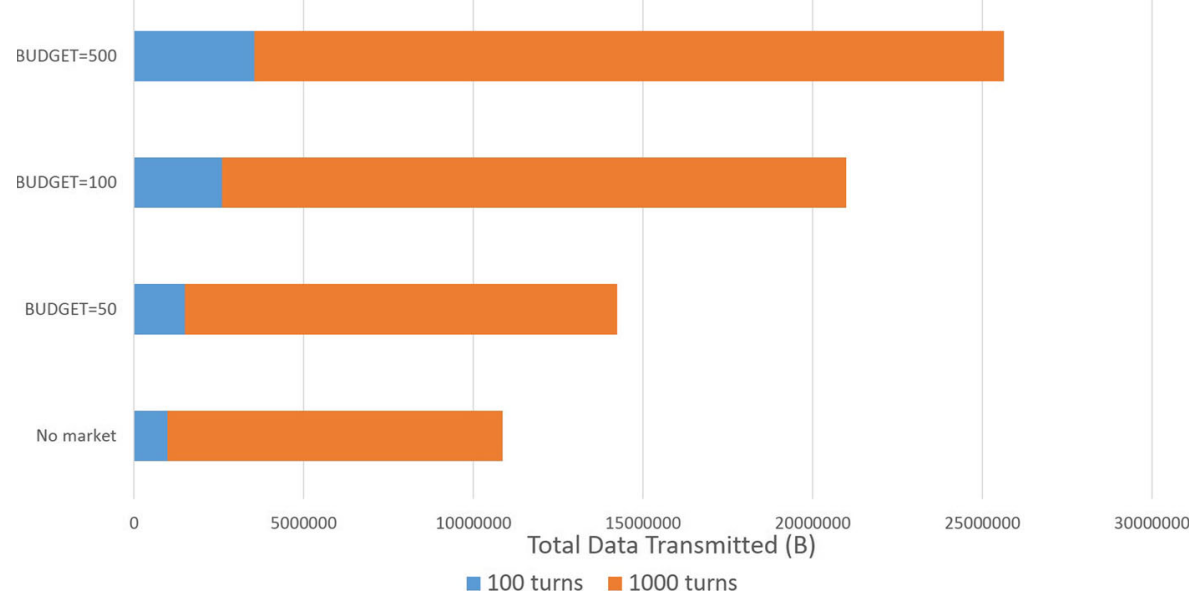

Fig. 1 Total Data Transmitted with varying budgets

buyer does not find a seller, he has no option but to receive or transmit at its default bitrate.

A buyer can also decide to purchase bandwidth from multiple devices. In this case, more than one seller provide their bandwidth to a single device. This is useful when very high priority data has to be transmitted since this operation will be more expensive than the 1-1 transactions.

We conducted experiments varying the bandwidth allocated $B$, the number of devices $N$ and different budgets of currency $C$. As a baseline we use a non-market scenario which is when devices cannot purchase extra bandwidth and must use what is allocated. To measure efficiency we use the amount of data transmitted (measured in bytes) over the entire simulation. The number of iterations (time) we use is 1000 .

\subsection{Varying budget}

First we conduct experiments varying the initial budget $C$ that is available to use for the devices. We varied $C$ as 50, 100, 200 and 500. The number of devices was kept constant at 10000. Figure 1 provides the results for both 100 and 1000 iterations. The results demonstrate that the market scenarios outperform the baseline allocation significantly with increased data transmitted as the budget is raised. This is easy to understand as the increased budget will provide more opportunities to purchase bandwidth. The baseline system does not use a budget and relies on users to transmit using the allocated bandwidth only.

\subsection{Varying total bandwidth available}

Next we vary the initial bandwidth $B$ made available to the devices as 1000, 2000, 5000, 10000 and 20000. We observe (as shown in Fig. 2) that even in scenarios where the overall bandwidth is really low, the market allocation strategy outperforms the 


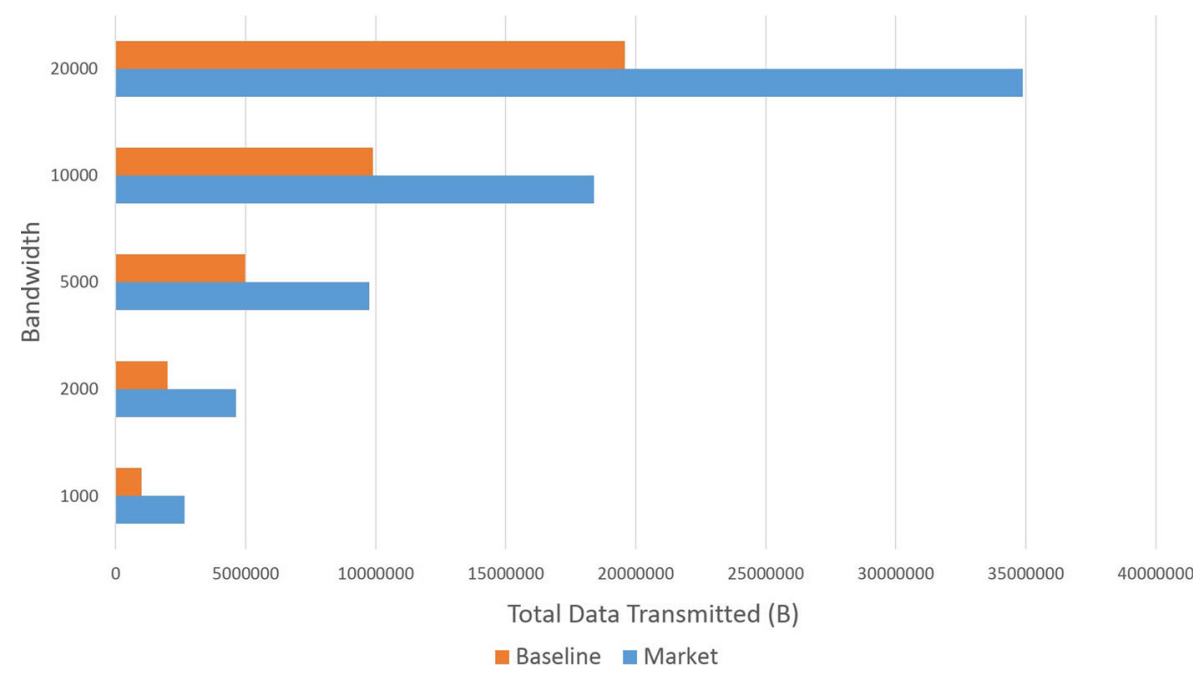

Fig. 2 Total Data Transmitted with varying total bandwidth

baseline significantly. The number of users was kept constant at 10000 and the initial budget at 100 for this experiment.

\subsection{Varying number of user devices}

Finally, we vary the number of user devices in the simulation over 1000, 2000, 5000, 10000 and 20000. In each case the initial budget $C$ was maintained at 100 and the initial bandwidth was kept at 10000. The results, as shown in Fig. 3 show that the

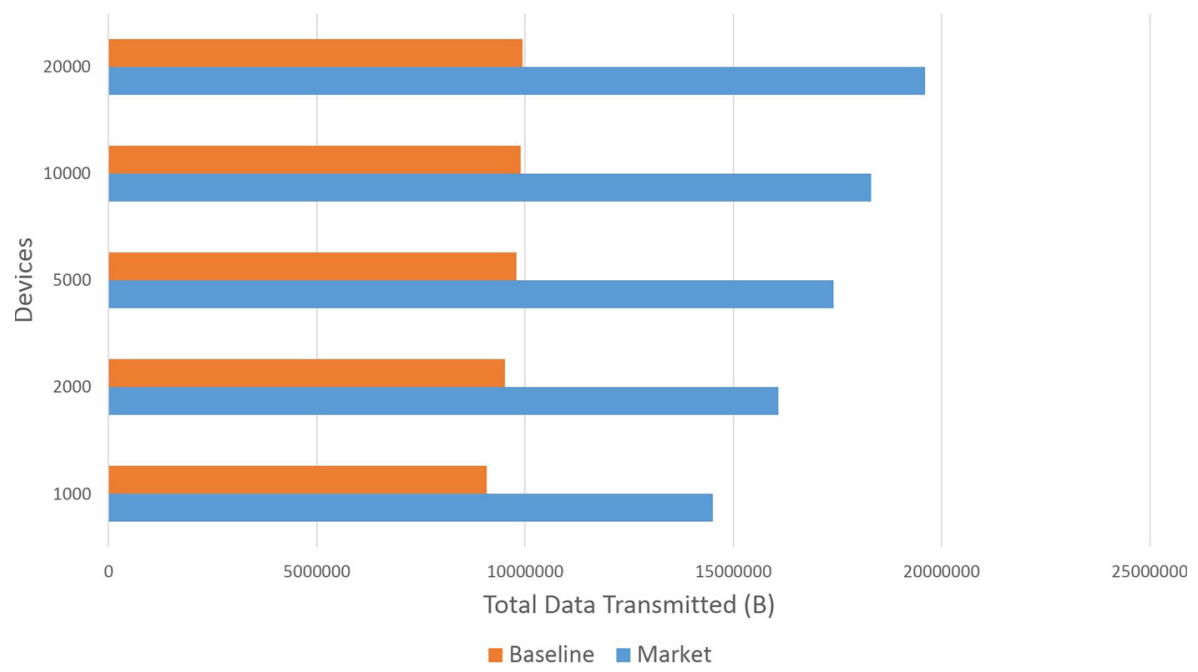

Fig. 3 Total Data Transmitted with varying number of user devices 


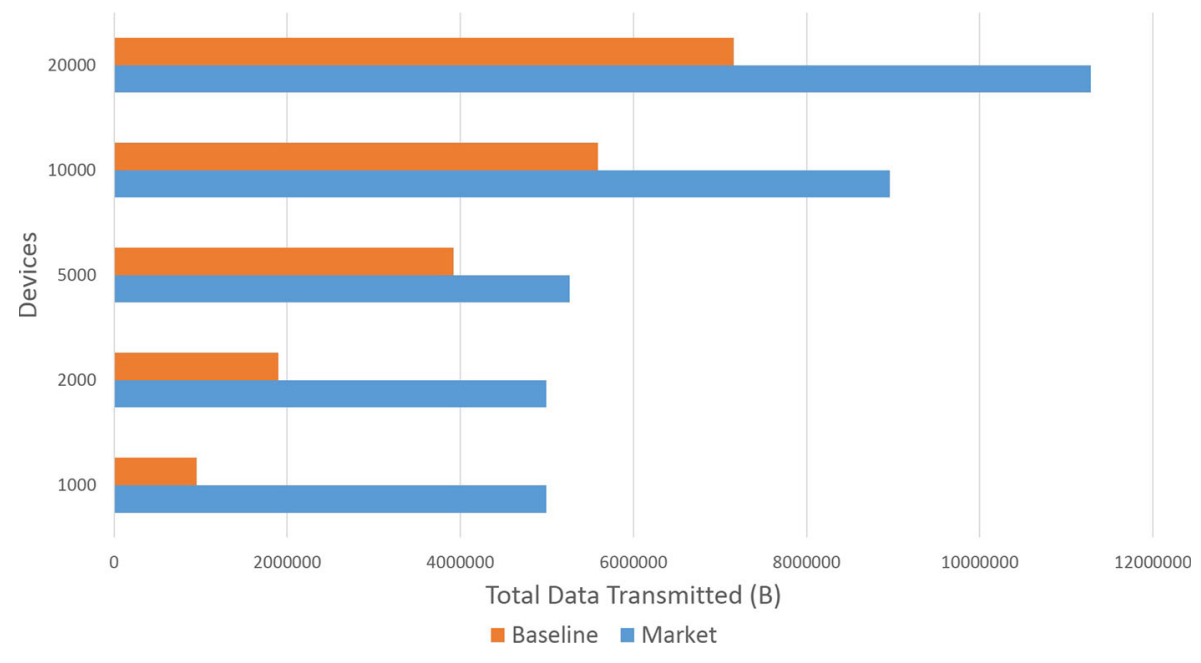

Fig. 4 Total Data Transmitted with small file sizes

data transmitted increases with the number of devices. This is due to the fact that as the number increases more devices are likely to sell bandwidth. On the other hand, and equally important, the baseline does not increase much because the number of devices is increased.

\subsection{Varying file sizes}

We also varied the size of the files. Earlier experiments are with large files simulating video streams. We also conducted an experiment with small files represented as a Gaussian with mean 1.9 and standard deviation 0.5. The results are shown in Fig. 4 and again we find the market method providing significant improvements in data transfer.

\section{Conclusions}

In this paper we have presented BidPacket, a market for trading bandwidth in public spaces. The market is simple to run and can operate above existing network protocols. Our experiments have shown that having such a trading market can significantly improve efficiency and provide a high rate of data transmission even in scenarios with low overall values of provisioned bandwidth or increased numbers of users. Thus Bidpacket can provide savings by reducing the amount of wifi they need to provision in public spaces such as hotel lobbies, airport terminals or trains.

The market also allows users to gain virtual currency for sharing their bandwidth. These earnings can be traded or substituted for real-world rewards such as hotel loyalty points, airline miles or coupons for products. 
Note that in the market mechanism described in this paper, we have used absolute values of required bandwidth. In a deployment scenario, a simpler setting could offer two levels of bandwidth access - standard and elite with users having the option of purchasing the higher level through trading with other customers.

Finally we point out that while in this paper we described the market as being used by individual users, in industrial settings it can be easily translated to wifi-enabled industrial devices such as sensors that need to transmit readings while conserving their resources such as power. These smart sensors can be easily programmed to bid for the necessary bandwidth when needed, and use their utility functions to request those required resources with the right amount of currency.

In future work, we plan to investigate a number of interesting scenarios, such as collusion among users, hoarding of resources for future use, and even handoffs from licensed spectrum to wifi.

Open Access This article is distributed under the terms of the Creative Commons Attribution 4.0 International License (http://creativecommons.org/licenses/by/4.0/), which permits unrestricted use, distribution, and reproduction in any medium, provided you give appropriate credit to the original author(s) and the source, provide a link to the Creative Commons license, and indicate if changes were made.

\section{References}

1. Clearwater, S.H. (1996). Market-based control: a paradigm for distributed resource allocation: World Scientific.

2. Covell, M., Baluja, S., \& Sukthankar, R. (2015). Micro-Auction Based Traffic-Light Control: Responsive, Local Decision Making. In IEEE Intelligent Transportation Systems Conference.

3. Guenther, O., Hogg, T., \& Huberman, B.A. (1998). Pattern Control in Unstable Structures. In In SPIE Conference on Smart Structures and Materials.

4. Huang, J., Berry, R., \& Honig, M. (2006). Auction-Based Spectrum sharing. In Mobile Networks and Applications, Vol. 11.

5. Huberman, B.A., \& Clearwater, S. (2005). A Multi-Agent System for Controlling Building Environments. In Proceedings of First International Conference on Multiagent Systems.

6. Lai, K., Rasmusson, L., Adar, E., Zhang, L., \& Huberman, B.A. (2005). Tycoon: An implementation of a distributed, market-based resource allocation system. In Multiagent and Grid Systems.

7. Lynch, J.P., \& Law, K.H. (2004). Decentralized energy market-based structural control. In Structural Engineering and Mechanics, Vol. 17.

8. Papavasiliou, A., Hindi, H., \& Greene, D. (2010). Market-based control mechanisms for electric power demand response. In 49th IEEE Conference on Decision and Control, CDC.

9. Paris, S., Martignon, F., Filippini, I., \& Chen, L. (2013). A bandwidth trading marketplace for mobile data offloading. In the Proceedings of INFOCOM, pp. 430-434: IEEE.

10. Qiu, Y., \& Marbach, P. (2003). Bandwidth Allocation in Ad Hoc Networks: A Price-Based Approach. In IEEE INFOCOM.

11. Rasmusson, L., \& Aurell, E. (2001). A Price Dynamics in Bandwidth Markets for Point-to-Point Connections. arXiv: cs/0102011.

12. Waldspurger, C.A., Hogg, T., Huberman, B.A., Kephart, J.O., \& Stornetta, W.S. (1992). Spawn: a distributed computational economy. In IEEE Transactions on Software Engineering 18.2: 103-117.

13. Wang, W., \& Baochun, L. (2005). Market-driven bandwidth allocation in selfish overlay networks. In the Proceedings of INFOCOM, 24th Annual Joint Conference of the IEEE Computer and Communications Societies. Proceedings IEEE, Vol. 4: IEEE. 